\title{
A robustness check procedure for hypofractionated Gamma Knife radiosurgery
}

\author{
Joshua Chiu, BSc, ${ }^{1}$ Steve Braunstein, MD, PhD, ${ }^{2}$ Jean Nakamura, MD, ${ }^{2}$ \\ Philip Theodosopoulos, MD, ${ }^{3}$ Penny Sneed, MD, ${ }^{2}$ Michael McDermott, MD, ${ }^{2}$ and Lijun Ma, PhD ${ }^{2}$ \\ ${ }^{1}$ University of Arizona College of Medicine, Tucson, Arizona; and Departments of ${ }^{2}$ Radiation Oncology and ${ }^{3}$ Neurological Surgery, \\ University of California, San Francisco, California
}

\begin{abstract}
OBJECTIVE Interfractional residual patient shifts are often observed during the delivery of hypofractionated brain radiosurgery. In this study, the authors developed a robustness treatment planning check procedure to assess the dosimetric effects of residual target shifts on hypofractionated Gamma Knife radiosurgery (GKRS).

METHODS The residual patient shifts were determined during the simulation process immediately after patient immobilization. To mimic incorporation of residual target shifts during treatment delivery, a quality assurance procedure was developed to sample and shift individual shots according to the residual uncertainties in the prescribed treatment plan. This procedure was tested and demonstrated for 10 hypofractionated GKRS cases.
\end{abstract}

RESULTS The maximum residual target shifts were less than $1 \mathrm{~mm}$ for the studied cases. When incorporating such shifts, the target coverage varied by $1.9 \% \pm 2.2 \%$ (range $0.0 \%-7.1 \%$ ) and selectivity varied by $3.6 \% \pm 2.5 \%$ (range $1.1 \%-9.3 \%$ ). Furthermore, when incorporating extra random shifts on the order of $0.5 \mathrm{~mm}$, the target coverage decreased by as much as $7 \%$, and nonisocentric variation in the dose distributions was noted for the studied cases.

CONCLUSIONS A pretreatment robustness check procedure was developed and demonstrated for hypofractionated GKRS. Further studies are underway to implement this procedure to assess maximum tolerance levels for individual patient cases.

https://thejns.org/doi/abs/10.3171/2018.7.GKS181581

KEYWORDS hypofractionation; Gamma Knife; stereotactic radiosurgery; treatment planning; surgical technique

$\mathrm{H}$ YPOFRACTIONATED brain radiosurgery has been widely implemented for managing brain lesions. ${ }^{2,3}$, 8,12 The goal of hypofractionation is to leverage tumor reoxygenation and normal tissue repair functions to enable a high dose of radiation to be delivered to the target while limiting normal tissue toxicities. ${ }^{10} \mathrm{~A}$ recent study investigating hypofractionated brain radiosurgery for treating large brain metastases ${ }^{7}$ found that hypofractionation significantly improved the tumor local control while reducing the rate of symptomatic radiation necrosis by as much as $10 \%$ or more. At our institution, we have applied hypofractionated Gamma Knife radiosurgery (GKRS) for treating large brain tumors, where normal tissue dose tol- erance significantly limits the dose that can be delivered to the target in a single fraction. ${ }^{6}$

One of the key features in hypofractionated GKRSbased treatments is the utilization of multiple isocenters $(n>10)$ for the purpose of covering a large target volume and, as such, the treatment typically lasts $10-30$ minutes. Because of noninvasive patient immobilization, target shifts are often observed during the treatment delivery. In this study, we studied the residual target shifts, which are defined as the target shifts immediately after patient setup or repeat setup. Because these are uncorrected shifts that would not trigger beam-off or stoppage of treatment, the question therefore arises as to how such residual shifts 
TABLE 1. Summary of the example cases tested for the robustness check procedures

\begin{tabular}{clccrrrr}
\hline Case No. & Indication & $\mathrm{D} / \mathrm{Fx}$ & $\mathrm{N}_{\text {iso }}$ & \multicolumn{1}{c}{ Vol $(\mathrm{ml})$} & \multicolumn{1}{c}{$\Delta \mathrm{x}(\mathrm{mm})$} & \multicolumn{1}{c}{$\Delta \mathrm{y}(\mathrm{mm})$} & \multicolumn{1}{c}{$\Delta \mathrm{z}(\mathrm{mm})$} \\
\hline 1 & VS & $20 / 5$ & 22 & 1.81 & $-0.20 \pm 0.51$ & $0.44 \pm 0.20$ & $0.16 \pm 0.19$ \\
\hline 2 & VS & $25 / 5$ & 20 & 1.34 & $0.34 \pm 0.53$ & $0.01 \pm 0.57$ & $0.20 \pm 0.44$ \\
\hline 3 & Metastasis & $24 / 3$ & 12 & 8.89 & $0.10 \pm 0.19$ & $0.05 \pm 0.23$ & $0.22 \pm 0.13$ \\
\hline 4 & Meningioma & $25 / 5$ & 15 & 4.65 & $0.09 \pm 0.16$ & $-0.11 \pm 0.25$ & $-0.22 \pm 0.15$ \\
\hline 5 & Glioma & $20 / 5$ & 27 & 18.38 & $0.13 \pm 0.63$ & $0.23 \pm 0.51$ & $0.33 \pm 0.71$ \\
\hline 6 & Meningioma & $25 / 5$ & 11 & 8.52 & $0.13 \pm 0.39$ & $-0.01 \pm 0.25$ & $0.34 \pm 0.19$ \\
\hline 7 & Metastasis & $30 / 5$ & 15 & 12.12 & $0.16 \pm 0.73$ & $0.41 \pm 0.35$ & $0.01 \pm 0.72$ \\
\hline 8 & Metastasis & $24 / 4$ & 25 & 17.14 & $-0.13 \pm 0.75$ & $-0.85 \pm 0.05$ & $-0.87 \pm 0.26$ \\
\hline 9 & Metastasis & $30 / 5$ & 16 & 8.02 & $0.11 \pm 0.22$ & $0.06 \pm 0.24$ & $0.10 \pm 0.36$ \\
\hline 10 & VS & $25 / 5$ & 24 & 5.56 & $0.29 \pm 0.35$ & $0.44 \pm 0.26$ & $-0.40 \pm 0.17$ \\
\hline
\end{tabular}

$\mathrm{D} / \mathrm{Fx}=$ dose per fraction; $\mathrm{N}_{\text {iso }}=$ total number of isocenters; $\mathrm{Vol}$ = target volume; $\mathrm{VS}$ = vestibular schwannoma.

might impact the delivered dose, and what are the acceptable maximum tolerance levels for monitoring such shifts, especially when multiisocenters are involved for hypofractionated GKRS treatments.

To address these questions, we developed a robustness check procedure and tested the procedure for 10 hypofractionated GKRS treatments. The primary goal of our study was to verify proper target coverage in the presence of the residual target shifts.

\section{Methods}

For robustness checks, the mean and standard deviations of the residual target shifts along $\mathrm{x}-, \mathrm{y}-$, and $\mathrm{z}$-axes were first measured during the patient simulation process when the patient was positioned on the treatment couch for a dry-run verification. These measurements were performed with either a digital probe, as described in our previous publication, ${ }^{6}$ or with an online high-definition motion management system of the Gamma Knife Icon system. ${ }^{15}$ The same measurements were also performed during the patient setup or repeat setup before a beam-on treatment delivery. Based on such measurements, each shot in the patient's treatment plan was then shifted according to the normal distribution of the same mean and standard deviations as measured. This was performed by first generating a series $(n=100)$ of random shift vectors $\left(\Delta x_{i}\right.$, $\Delta \mathrm{y}_{\mathrm{i}}, \Delta \mathrm{z}_{\mathrm{i}}$ ) by sampling the normal distributions along the $\mathrm{x}-$, $y$-, or z-direction for the ith shot. The coordinates for the ith shot $\left(x_{i}, y_{i}, z_{i}\right)$ were then set to $\left(x_{i}+\Delta x_{i}, y_{i}+\Delta y_{i}, z_{i}+\Delta z_{i}\right)$. The coordinates of these individually shifted shots were finally imported back into the clinical planning system for dose recomputations. Note that the beam-on time of each shot was listed in the current Leksell GammaPlan system for the delivery of individual treatment fraction. Therefore residual target shifts were simulated for intrafractional effects of a single fraction delivery of the treatment course.

In order to construct such a dose calculation within the clinical treatment planning system (LGP version 11.1), an iterative procedure was developed. This is because the current system is configured to automatically renormalize the dose distribution according to the point maximum dose inside the target, and any shifts in the shot coordinates would alter the beam-on time of each shot. To en- sure individual shot beam-on time remained unchanged to mimic the actual treatment delivery, the relative weights of each shots were scaled via the procedure as follows: 1) Import the residual shifts sampled shot coordinates into the LGP planning system. 2) Compute the beam-on time for individual shots $T_{i}$. 3) Set the beam weight of each shifted shot to $\mathrm{W}_{\mathrm{i}}=100 \times \mathrm{W}_{0, \mathrm{i}} \times \mathrm{T}_{0, \mathrm{i}} / \mathrm{T}_{\mathrm{i}}$, where $\mathrm{W}_{0, \mathrm{i}}$ is the ith shot weight of the original plan and $\mathrm{T}_{0, \mathrm{i}}$ is the beam-on time for the ith shot for the original plan. The multiplication by a factor of 100 is to minimize the rounding error. 4) Scale the prescription dose to the new value $D=D_{0} \times$ $\mathrm{T}_{\text {total }} / \mathrm{ST}_{\text {total }}$, where $\mathrm{T}_{\text {total }}$ is the total beam-on time for the original plan and $\mathrm{ST}_{\text {total }}$ is the total beam-on time for the sampled treatment plan. 5) Go to step 3 until the resulting beam-on time for each shot agree with $\mathrm{T}_{\mathrm{i}, 0}$ of the original plan within 0.01 minutes.

For example, suppose an 8-mm shot in the original treatment plan carried a relative weighting of 1.00 for a beam-on time of 2.4 minutes. After introducing residual target motions to all the shots, this $8-\mathrm{mm}$ shot yielded a beam-on time of 2.0 minutes. To reconstruct the original treatment time, the relative weighting for this shot therefore should be set to $100 \times 2.4 / 2.0=120$ instead. Once all the shots were scaled, the prescription dose for the residual shifts sampled plan was readjusted to match the total beam-on times of the original treatment plan. In general, with one to two iterations the beam-on time in each shot was restored to the original plan within $0.01 \mathrm{~min}$. The underlying assumption of the above procedure is that any shifts introduced from residual target motions would be negligible in altering the value of absolute dose rate at the center of the shot. This is generally valid for our cases because the maximum residual target shifts are small (i.e., within $1.5 \mathrm{~mm}$ for all the cases).

\section{Results}

The hypofractionated brain radiosurgery procedures are summarized in Table 1 . The average number of isocenters $( \pm$ SD) used for these cases was $19 \pm 6$ (range 11-27). The means and SDs of the measured residual target shifts are summarized in Table 1 . The mean residual target shifts were $\delta_{\mathrm{x}}=0.10 \pm 0.45 \mathrm{~mm}$ (lateral direction), $\delta_{\mathrm{y}}=0.09 \pm$ $0.40 \mathrm{~mm}$ (anterior-posterior direction), and $\delta_{\mathrm{z}}=0.01 \pm$ 


\begin{tabular}{|c|c|c|c|c|c|c|c|c|c|c|c|c|c|c|c|}
\hline \multicolumn{15}{|c|}{ Shot summary } & 토 \\
\hline Shots & $\begin{array}{l}X \\
{[\mathrm{~mm}]}\end{array}$ & $\begin{array}{l}\mathrm{Y} \\
{[\mathrm{mm}]}\end{array}$ & $\begin{array}{l}\mathrm{Z} \\
{[\mathrm{mm}]}\end{array}$ & $\begin{array}{l}\text { Gamma } \\
\text { [deg] }\end{array}$ & Weight & Colli & ators & & & & & & & $\begin{array}{l}\text { Time } \\
\text { [min] }\end{array}$ & \\
\hline 1 & 120.5 & 69.1 & 127.6 & 90 & 130.00 & 8 & 8 & 8 & 8 & 8 & 8 & 8 & 8 & 1.99 & \\
\hline 2 & 127.1 & 85.1 & 134.4 & 90 & 110.00 & 4 & B & B & 8 & 8 & 8 & 8 & 8 & 2.45 & \\
\hline 3 & 123.9 & 75.2 & 134.8 & 90 & 60.00 & 4 & 4 & 4 & 4 & 4 & 4 & 4 & 4 & 1.00 & \\
\hline 4 & 132.9 & 86.9 & 129.5 & 90 & 160.00 & 4 & 4 & 4 & B & B & B & 4 & 4 & 4.13 & \\
\hline 5 & 132.7 & 85.6 & 126.0 & 90 & 100.00 & 4 & 4 & 4 & 4 & 4 & 4 & 4 & 4 & 1.64 & \\
\hline 6 & 117.9 & 85.9 & 120.4 & 90 & 100.00 & 8 & 8 & 8 & 8 & 8 & 8 & 8 & 8 & 1.54 & \\
\hline 7 & 122.1 & 77.8 & 120.7 & 90 & 90.00 & 4 & 8 & 8 & 8 & 8 & 4 & 4 & 4 & 1.43 & \\
\hline 8 & 119.2 & 69.0 & 121.4 & 90 & 110.00 & 8 & 8 & 8 & 4 & 8 & 8 & 8 & 8 & 1.70 & \\
\hline 9 & 114.1 & 82.6 & 115.3 & 90 & 30.00 & 4 & 4 & 4 & 4 & 4 & 4 & 4 & 4 & 0.51 & \\
\hline 10 & 109.2 & 78.0 & 126.3 & 90 & 80.00 & 4 & 4 & B & 4 & 4 & 4 & 4 & 4 & 1.59 & \\
\hline 11 & 111.3 & 84.0 & 124.3 & 90 & 50.00 & 8 & 8 & 8 & 8 & 8 & 8 & 8 & 8 & 0.78 & \\
\hline 12 & 123.6 & 75.2 & 129.4 & 90 & 100.00 & 8 & 8 & 8 & 8 & 8 & 8 & 8 & 8 & 1.53 & \\
\hline 13 & 114.5 & 87.2 & 124.3 & 90 & 100.00 & 4 & 4 & 4 & 4 & 4 & 4 & 4 & 4 & 1.70 & \\
\hline 14 & 110.7 & 77.3 & 121.6 & 90 & 100.00 & 8 & 8 & B & 8 & 8 & 4 & B & 4 & 2.17 & \\
\hline 15 & 112.6 & 86.2 & 119.6 & 90 & 60.00 & 8 & 4 & 4 & 8 & 8 & 4 & 4 & 8 & 0.97 & \\
\hline 16 & 111.1 & 79.4 & 118.4 & 90 & 100.00 & 4 & 4 & 4 & 4 & 4 & 4 & 4 & 4 & 1.70 & \\
\hline 17 & 128.2 & 82.2 & 127.4 & 90 & 100.00 & 8 & 8 & 8 & 8 & 8 & 8 & 8 & 8 & 1.51 & \\
\hline 18 & 126.1 & 86.8 & 124.5 & 90 & 99.90 & 8 & 8 & 8 & 8 & 8 & 8 & 8 & 8 & 1.52 & \\
\hline 19 & 114.3 & 75.9 & 127.9 & 90 & 109.90 & 8 & 8 & 8 & 8 & 8 & 8 & 8 & 8 & 1.71 & \\
\hline 20 & 117.3 & 76.3 & 118.2 & 90 & 120.00 & 8 & 8 & 8 & 8 & 8 & 8 & 8 & 8 & 1.84 & \\
\hline 21 & 114.8 & 83.2 & 114.6 & 90 & 58.00 & 8 & 8 & 8 & 8 & 8 & 8 & 8 & 8 & 0.89 & \\
\hline 22 & 129.5 & 79.0 & 122.1 & 90 & 80.00 & 8 & 8 & 4 & 8 & 8 & 8 & 8 & 8 & 1.22 & \\
\hline 23 & 114.0 & 83.5 & 127.7 & 90 & 120.00 & 8 & 8 & 8 & 8 & 8 & 8 & 8 & 8 & 1.87 & \\
\hline \multirow[t]{2}{*}{24} & 120.5 & 78.2 & 132.7 & 90 & 130.00 & 8 & 8 & 8 & 8 & 8 & 8 & 8 & 8 & 2.01 & \\
\hline & & & & & & & & & & & \multicolumn{3}{|c|}{ Close } & Helelp & \\
\hline
\end{tabular}

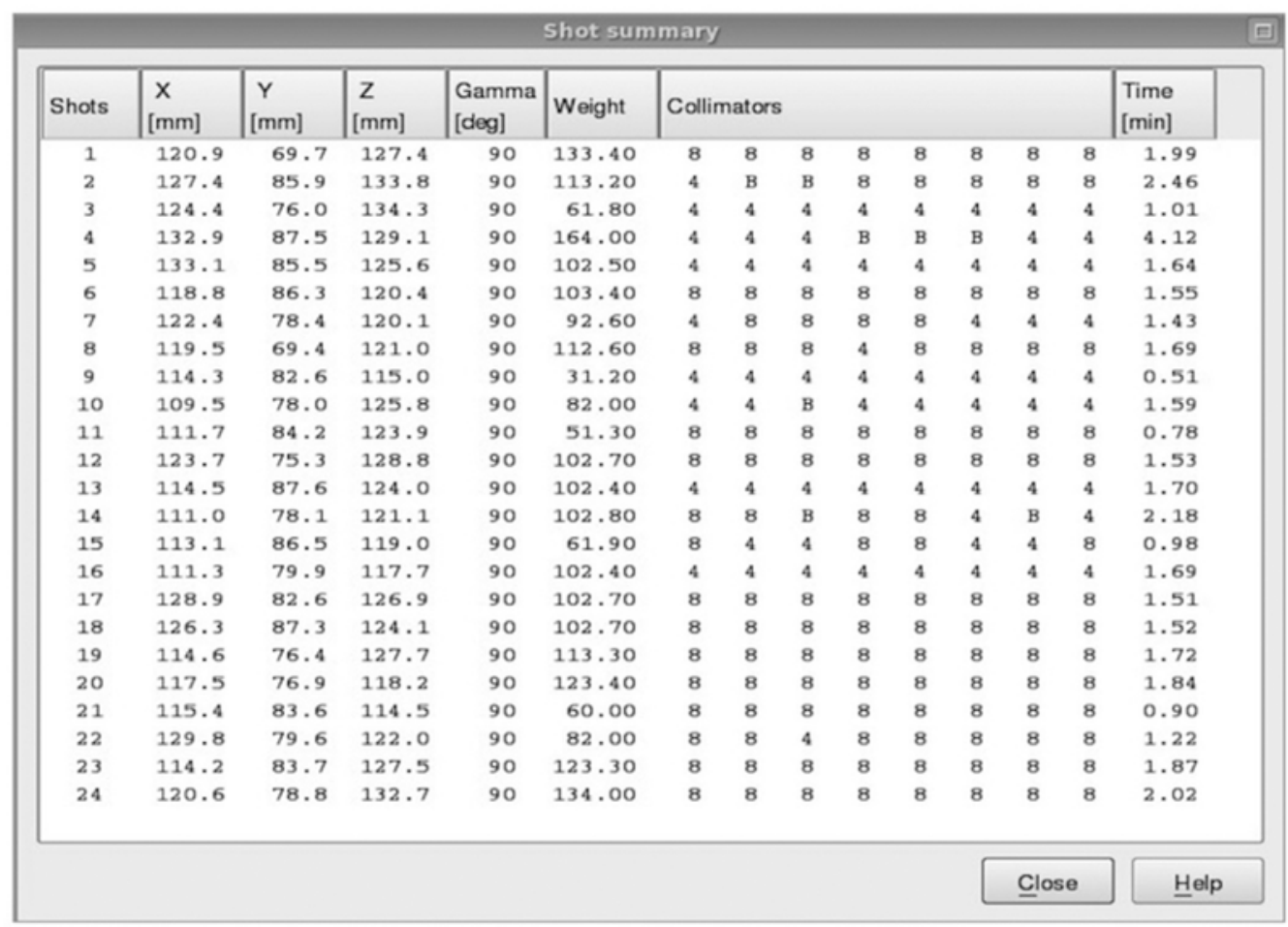

FIG. 1. Results of an example case illustrating the procedure adding the residual shifts to each shot while maintaining the beam-on time of individual shots: shot summary for the prescribed treatment plan (upper) and shot summary for the residual shifts sampled treatment plan (lower). The total beam-on time and individual shot irradiation time matched the original plan to within 0.01 minutes.

$0.33 \mathrm{~mm}$ (superior-inferior direction). An illustration of the individual shot-simulation procedure is illustrated in Fig. 1, where the shot time agreed with the original treatment plan within 0.01 minutes from the procedure
The isodose distribution comparison for case 10 of Table 1 is shown in Fig. 2. A small difference $(<3 \%)$ between the residual error-sampled dose distribution (Fig. 2B) and the original treatment plan (Fig. 2A) was found. However, 

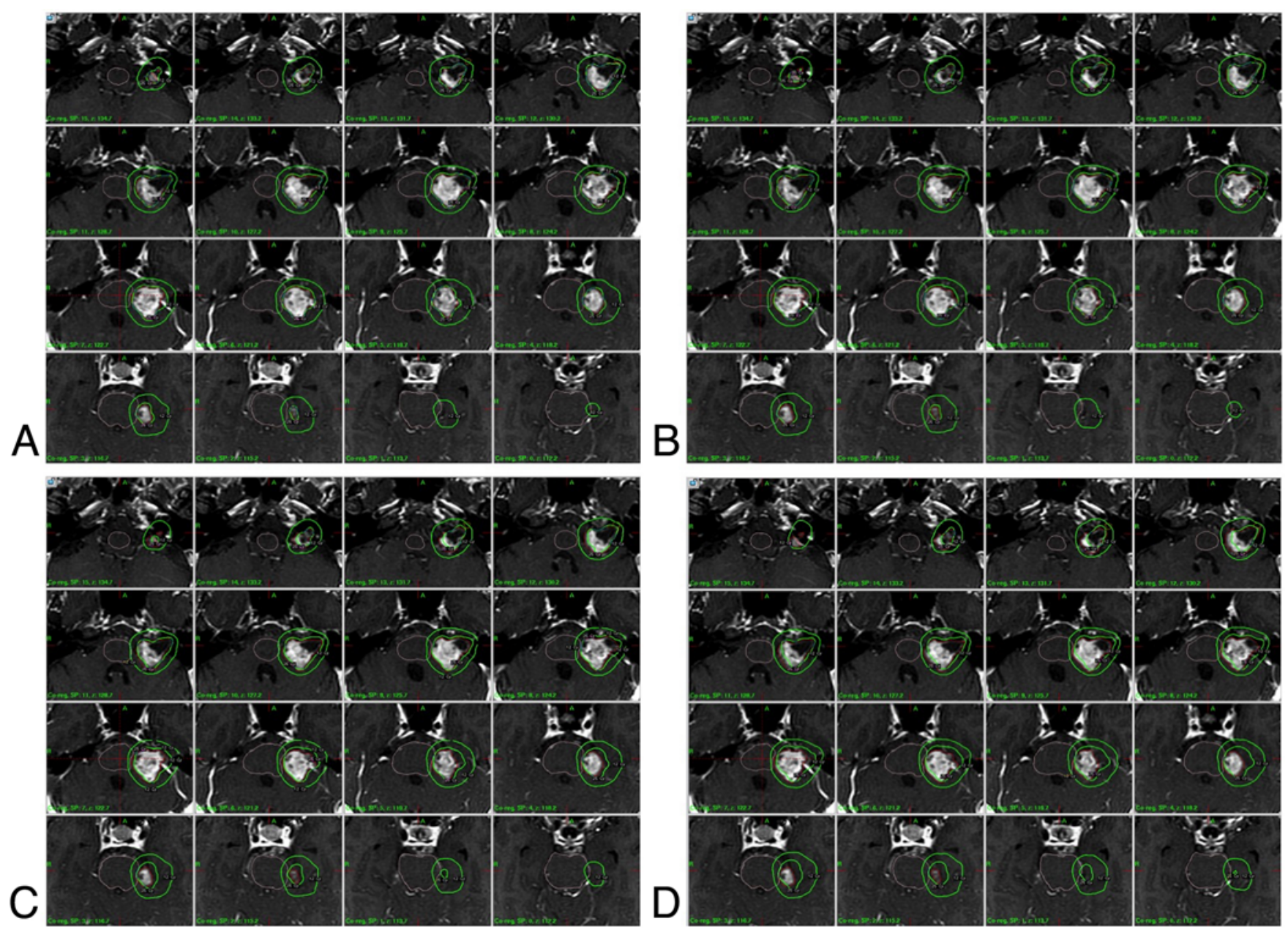

FIG. 2. Axial isodose distributions of the example case: the prescribed treatment plan $(\mathbf{A})$, treatment plan incorporating the measured residual shifts of $\Delta x=0.29 \pm 0.35 \mathrm{~mm}, \Delta y=0.44 \pm 0.26 \mathrm{~mm}, \Delta z=-0.40 \pm 0.17$ (B), the isodose distribution where additional random shifts of $0.2 \mathrm{~mm}$ were added to the measured residual shifts along each direction $(\mathbf{C})$, and the isodose distribution where additional random shifts of $0.4 \mathrm{~mm}$ were added to each direction (D).

if additional random shifts of $0.2 \mathrm{~mm}$ (Fig. 2C) and 0.4 $\mathrm{mm}$ (Fig. 2D) were added to the residual shifts, significant differences $(>5 \%)$ in the dose distributions were noted, especially near the most superior and inferior portion of the target. Variations in the dose-volume histogram for the dose matrix region encompassing the target are also plotted in Fig. 3. In contrast to the target dose-volume histogram, a significantly smaller variation $(<5 \%)$ was noted for the dose matrix, indicating general conservation of the isodose volumes near the prescription doses.

Finally, a summary of the target coverage and selectivity variation for all the cases is plotted in Fig. 4. On average, residual shifts decreased the mean target coverage from $98.5 \% \pm 1.0 \%$ to $96.7 \% \pm 1.9 \%$ in the target coverage and the selectivity decreased from $0.78 \pm 0.11$ to $0.75 \pm 0.11$ in selectivity for all the cases. Since the Paddick conformity index is defined as the product of selectivity and target coverage, the maximum decrease in Paddick conformity index was therefore $<5 \%$ based on the observed target coverage and selectivity variations. Furthermore, the maximum variation in the dose gradient index (derived from the volume ratio of two dose values on the dose-volume histogram curve of the calculation dose matrix) was also $<5 \%$ for the studied cases. However, if an extra $0.4 \mathrm{~mm}$ were introduced, the target coverage decreased to $94.3 \% \pm$ $2.2 \%$ and selectivity decreased to $0.72 \pm 0.09$ instead. As a result, the constraint of maintaining at least $95 \%$ coverage of the target volume cannot be satisfied for 7 of 10 treatments with residual shifts of $0.4 \mathrm{~mm}$.

\section{Discussion}

In this technical note, we report a simple procedure to assess the robustness of a treatment plan against residual target shifts for GKRS. The procedure was implemented for a cohort of hypofractionated Gamma Knife radiosurgery cases $(n=10)$. The residual target shifts were measured to be submillimeters and they were in agreement with the previous studies for the performance of the Gamma Knife eXtend and the Icon system. ${ }^{6,9,11,13}$

On average, the effect of the residual target shifts was found to be small $(<3 \%)$ in terms of target coverage and selectivity variability. However, when incorporating extra random shifts on the order of $0.5 \mathrm{~mm}$ to the residual tar- 

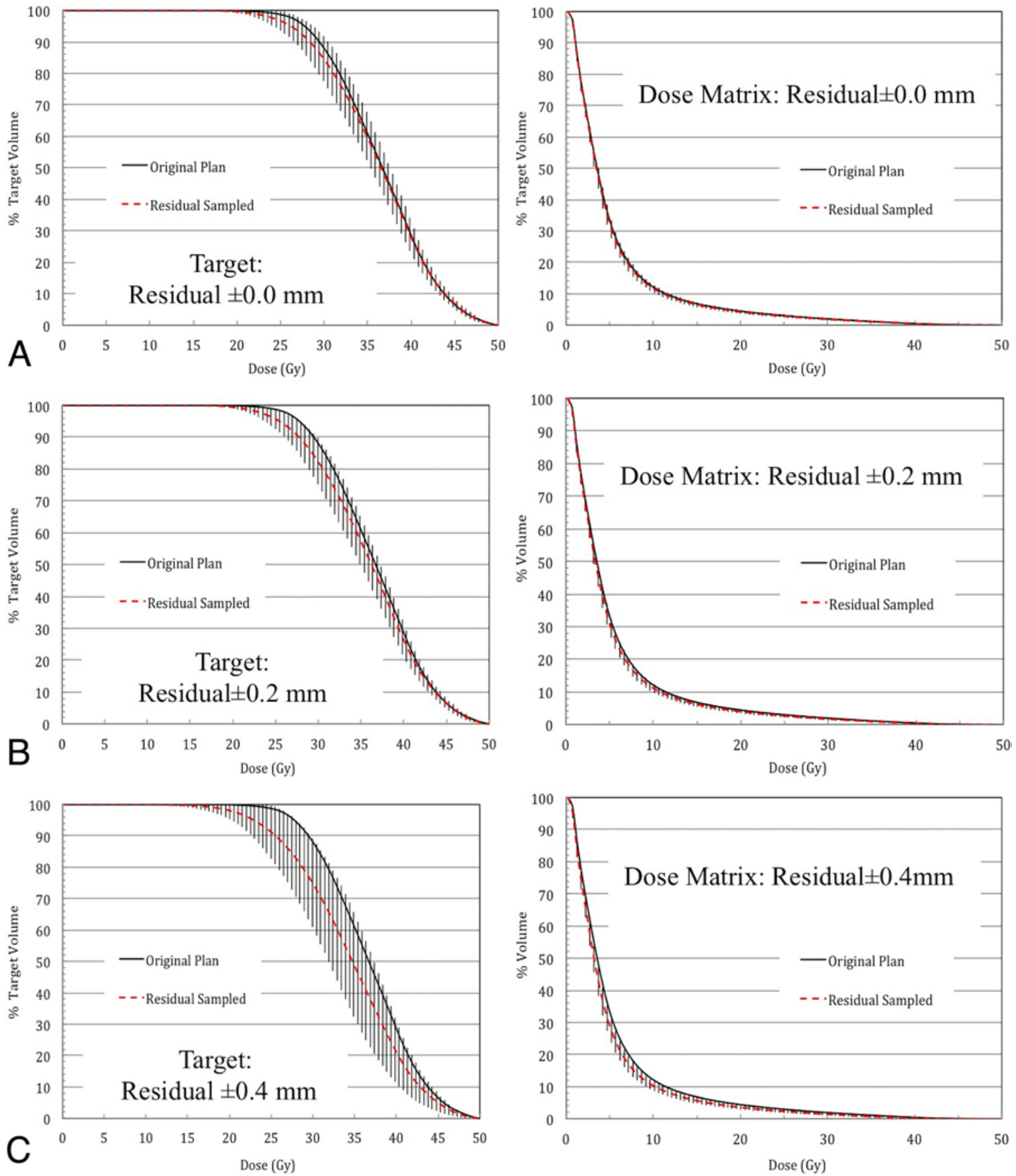

FIG. 3. Dose-volume histogram comparing the original treatment plan and the treatment plan incorporating measured residual shifts $(\mathbf{A})$, residual shifts plus random shifts of additional $0.2 \mathrm{~mm}$ along each direction $(\mathbf{B})$, and residual shifts plus random shifts of additional $0.4 \mathrm{~mm}$ along each direction (C). Note that the target coverage consistently decreased to $95 \%$ when additional $0.4-\mathrm{mm}$ shifts were added to the residual shifts, thus setting the maximum tolerance level for the treatment delivery.

get shifts, significant dosimetric effects were noted, and the procedure allowed us to evaluate the minimum intrafractional tolerance levels required for patient-specific treatments. This is because the residual target shifts by definition represent the best achievable targeting alignment following a treatment setup. These shifts served as the baseline value to assess additional target shifts so that patient-specific tolerance levels may be determined for the whole treatment delivery.
Due to conical radiation source arrangement of the current GK unit, the dose fall-off tends to be nonisotropici.e., the dose fall-off tends to be steeper along the longitudinal direction compared to the other directions. Combining such an effect with residual target shifts, variations in the final dose distribution are likely nonisotropic, as illustrated in the example case of Fig. 2. This suggests that patient-specific tolerance levels for maximum target shifts along certain directions may be needed when performing 

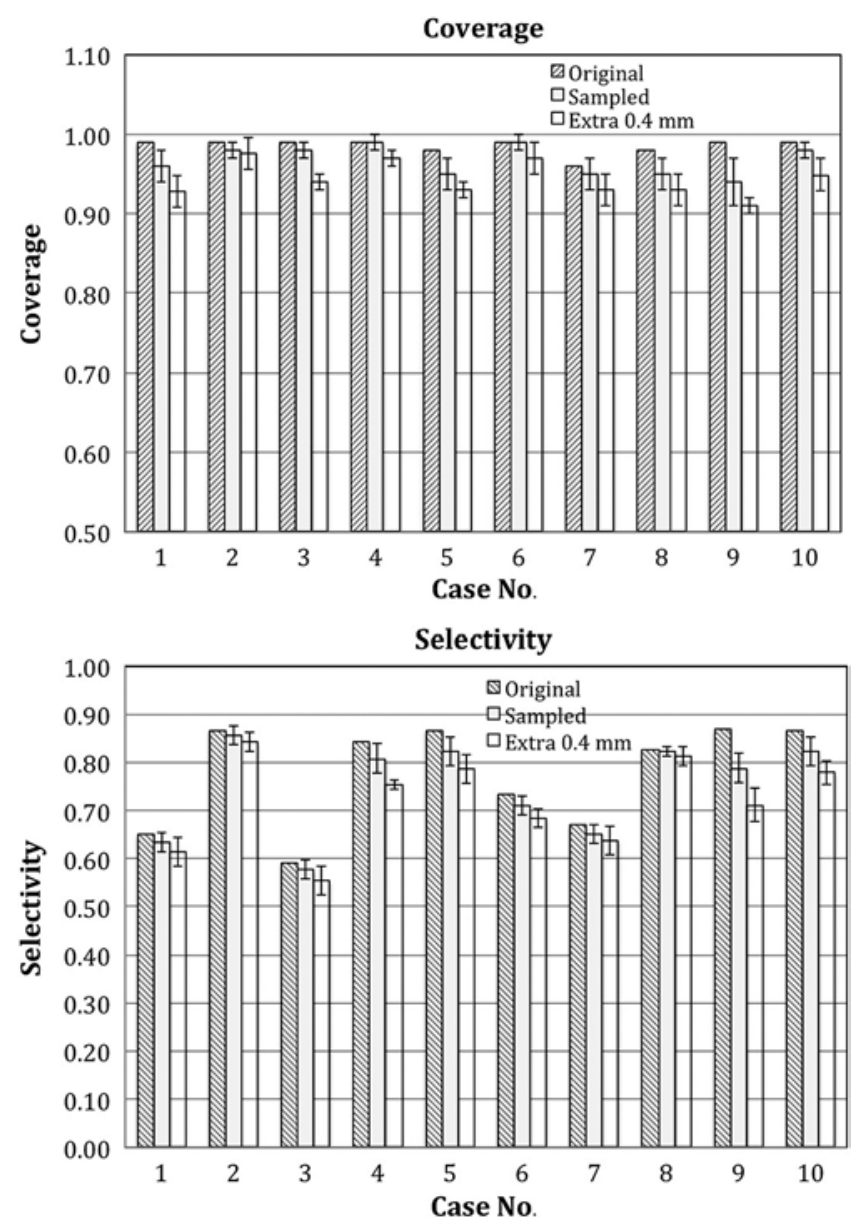

FIG. 4. Results of variations in the target coverage and selectivity due to the measured residual shifts for the studied 10 cases. The results of adding $0.4-\mathrm{mm}$ random shifts to the residual target shifts are also plotted for comparison.

hypofractionated GKRS treatment. Currently, only composite shifts from all directions are monitored during the hypofractionated treatments with the Gamma Knife Icon. Further studies are therefore needed to determine the pros and cons of implementing such a practice for individual patient treatments.

For the proof of concept and demonstration of the robustness check procedure, a small number of cases $(\mathrm{n}=$ 10) were investigated in this study. In theory, the procedure can be applied to other modalities such as linear accelerator (LINAC)-based radiosurgical treatments, where residual target shifts have been studied for both intracranial and extracranial sites. ${ }^{1,4,5,9,14}$ Since LINAC-based treatments tend to employ one or few isocenters, while GKRS employs a high number of isocenters $(>10)$, especially for treating a large target, the dose-averaging effect across different isocenters can be significantly different between GKRS and LINAC-based treatments. Future studies are therefore needed to quantify such differences and general applicability of the procedure.

In conclusion, a robustness planning check procedure was developed and demonstrated for hypofractionated GKRS. The procedure allows a user to evaluate dosimetric effects of residual target shifts, thus determining maximum target shifts allowed to ensure adequate target coverage when delivering a hypofractionated Gamma Knife treatment. Our ongoing study to apply this procedure to develop near-real time dose adaption with the latest Gamma Knife Icon system is aimed to deliver more precise hypofractionated treatments.

\section{References}

1. Badakhshi H, Barelkowski T, Wust P, Budach V, Boehmer D, Graf R: Intrafraction variations in linac-based image-guided radiosurgery of intracranial lesions. Cancer Radiother 17:664-667, 2013

2. Dong P, Pérez-Andújar A, Pinnaduwage D, Braunstein S, Theodosopoulos P, McDermott M, et al: Dosimetric characterization of hypofractionated Gamma Knife radiosurgery of large or complex brain tumors versus linear accelerator-based treatments. J Neurosurg 125 (Suppl 1):97-103, 2016

3. Eaton BR, LaRiviere MJ, Kim S, Prabhu RS, Patel K, Kandula $S$, et al: Hypofractionated radiosurgery has a better safety profile than single fraction radiosurgery for large resected brain metastases. J Neurooncol 123:103-111, 2015 (Erratum in J Neurooncol 123:113, 2015)

4. Guckenberger M, Roesch J, Baier K, Sweeney RA, Flentje M: Dosimetric consequences of translational and rotational errors in frame-less image-guided radiosurgery. Radiat Oncol 7:63, 2012

5. Li W, Sahgal A, Foote M, Millar BA, Jaffray DA, Letourneau $\mathrm{D}$ : Impact of immobilization on intrafraction motion for spine stereotactic body radiotherapy using cone beam computed tomography. Int J Radiat Oncol Biol Phys 84:520526, 2012

6. Ma L, Pinnaduwage D, McDermott M, Sneed PK: Wholeprocedural radiological accuracy for delivering multi-session Gamma Knife radiosurgery with a relocatable frame system. Technol Cancer Res Treat 13:403-408, 2014

7. Minniti G, Scaringi C, Paolini S, Lanzetta G, Romano A, Cicone F, et al: Single-fraction versus multifraction $(3 \times$ $9 \mathrm{~Gy})$ stereotactic radiosurgery for large $(>2 \mathrm{~cm})$ brain metastases: a comparative analysis of local control and risk of radiation-induced brain necrosis. Int J Radiat Oncol Biol Phys 95:1142-1148, 2016

8. Pessina F, Navarria P, Cozzi L, Ascolese AM, Maggi G, Riva $\mathrm{M}$, et al: Outcome evaluation of oligometastatic patients treated with surgical resection followed by hypofractionated stereotactic radiosurgery (HSRS) on the tumor bed, for single, large brain metastases. PLoS One 11:e0157869, 2016

9. Ruschin M, Nayebi N, Carlsson P, Brown K, Tamerou M, Li $\mathrm{W}$, et al: Performance of a novel repositioning head frame for gamma knife Perfexion and image-guided linac-based intracranial stereotactic radiotherapy. Int J Radiat Oncol Biol Phys 78:306-313, 2010

10. Sahgal A, Ma L, Chang E, Shiu A, Larson DA, Laperriere $\mathrm{N}$, et al: Advances in technology for intracranial stereotactic radiosurgery. Technol Cancer Res Treat 8:271-280, 2009

11. Schlesinger D, Xu Z, Taylor F, Yen CP, Sheehan J: Interfraction and intrafraction performance of the Gamma Knife Extend system for patient positioning and immobilization. $\mathbf{J}$ Neurosurg 117 Suppl:217-224, 2012

12. Scorsetti M, Facoetti A, Navarria P, Bignardi M, De Santis M, Ninone SA, et al: Hypofractionated stereotactic radiotherapy and radiosurgery for the treatment of patients with radioresistant brain metastases. Anticancer Res 29:4259-4263, 2009

13. Wright G, Harrold N, Hatfield P, Bownes P: Validity of the use of nose tip motion as a surrogate for intracranial motion in mask-fixated frameless Gamma Knife ${ }^{\circledR}$ Icon $^{\text {TM }}$ therapy. J Radiosurg SBRT 4:289-301, 2017 
14. Xie Y, Djajaputra D, King CR, Hossain S, Ma L, Xing L: Intrafractional motion of the prostate during hypofractionated radiotherapy. Int J Radiat Oncol Biol Phys 72:236-246, 2008

15. Zeverino M, Jaccard M, Patin D, Ryckx N, Marguet M, Tuleasca C, et al: Commissioning of the Leksell Gamma Knife ${ }^{\circledR}$ Icon $^{\mathrm{TM}}$. Med Phys 44:355-363, 2017

\section{Disclosures}

The authors report no conflict of interest concerning the materials or methods used in this study or the findings specified in this paper.

\section{Author Contributions}

Conception and design: Ma, Braunstein, Nakamura, Theodosopoulos, Sneed, McDermott. Acquisition of data: Ma, Chiu, McDermott. Analysis and interpretation of data: Ma, Chiu,
Braunstein, McDermott. Drafting the article: Ma, Chiu, Braunstein. Critically revising the article: Ma, Chiu, Braunstein, Nakamura, Sneed. Reviewed submitted version of manuscript: Ma, Chiu, Braunstein, Nakamura, Theodosopoulos, Sneed. Approved the final version of the manuscript on behalf of all authors: Ma. Administrative/technical/material support: McDermott. Study supervision: Ma.

\section{Supplemental Information}

\section{Previous Presentations}

Presented orally in part at the Leksell Gamma Knife Society Meeting, March 4-8, 2018, Dubai, United Arab Emirates.

\section{Correspondence}

Lijun Ma: University of California, San Francisco, CA. lijun.ma@ ucsf.edu. 